\title{
Role of Allelopathic Weed Management on Nutrient Uptake in Pigeonpea (Cajanus cajan L.)
}

\author{
Shridhara $^{1}$, B. Nagoli ${ }^{\text {* }}$, B. M. Dodamani ${ }^{2}$, M. Y. Ajaykumar ${ }^{2}$, \\ Pandit S. Rathod $^{3}$, Mahadeva Swamy ${ }^{4}$ and K. Basavaraj ${ }^{5}$ \\ ${ }^{1}$ College of Agriculture, Raichur, India \\ ${ }^{2}$ College of Agriculture, Kalaburagi, India \\ ${ }^{3}$ Main Agriculture Research Station, Raichur, India \\ ${ }^{4}$ Zonal Agriculture Research Station, Kalaburagi, India \\ ${ }^{5}$ University of Agriculture sciences, Raichur, India
}

\section{Keywords}

Allelopathy, Nutrient uptake, Grain yield

Article Info

Accepted:

12 December 2020 Available Online: 10 January 2021

\section{A B S T R A C T}

A pot experiment was carried out at Zonal Agriculture Research Station, Kalaburagi (Karnataka), during kharif 2018 and 2019 to study the role of allelopathic weed management on nutrient uptake in pigeonpea (Cajanus cajan L.). The soil was medium deep black with soil $\mathrm{pH}(8.13)$, EC $\left(0.25 \mathrm{dS} \mathrm{m}^{-1}\right)$, available nitrogen $\left(242 \mathrm{~kg} \mathrm{ha}^{-1}\right), \mathrm{P}_{2} \mathrm{O}_{5}$ $\left(34 \mathrm{~kg} \mathrm{ha}^{-1}\right)$ and $\mathrm{K}_{2} \mathrm{O}\left(347 \mathrm{~kg} \mathrm{ha}^{-1}\right)$. The experiment was laid out in completely randomised block design which comprised of 18 treatments with three replications, consisting four plant extracts, i.e., Sorghum, Parthenium, Cassia and Eucalyptus and imazythapyre herbicide. All the practices followed were according to package of practice of UAS, Raichur. The data of each crop season were statistically analyzed. Weed management practices significantly influenced the grain yield and nutrient uptake (N, P and K). Among all the treatments; Imazythapyre @ 56.25 g a.i./ha + sorghum plant extract at 20-25 DAS, imazythapyre@56.25 g a.i./ha + cassia plant extract at 20-25 DAS, imazythapyre @ $56.25 \mathrm{~g}$ a.i./ha + parthenium plant extract at 20-25 DAS, imazythapyre @ $37.5 \mathrm{~g}$ a.i./ha + sorghum plant extract at 20-25 DAS, imazythapyre @ $56.25 \mathrm{~g}$ a.i./ha + eucalyptus leaf extract at 20-25 DAS, imazythapyre @ 37.5 g a.i./ha + cassia plant extract at 20-25 DAS, imazythapyre@37.5 g a.i./ha + parthenium plant extract at 20-25 DAS and imazythapyre @ 37.5 g a.i./ha + eucalyptus leaf extract at 20-25 DAS recorded significantly higher grain yield and uptake of nitrogen, phosphorus and potassium compared to rest of the treatments. Plant extracts when used with herbicide help to reduce usage of herbicide by $50 \%$ by avoiding ill effects on soil fertility and productivity can be maintained for longer period.

\section{Introduction}

Pulses are an important component of agricultural food crops and the cheapest source of dietary protein. The high content of protein in pulses makes the diet more nutritive for vegetarian when consumed with other cooked food items. Pulses are rich source of protein, dietary fiber, complex carbohydrates and $\mathrm{K}, \mathrm{Se}, \mathrm{Fe}$ and $\mathrm{Zn}$-power house of 
nutrients. Pulses are also known for increasing productivity of soil through fixation of nitrogen from atmosphere. Pulses are well suited in rainfed condition and require less farm resources; hence farmers prefer to grow them from economic point of view throughout the country that helps to eliminate hunger, food security and malnutrition. India plays a key role in global pulse production and contributes about 25 per cent to the total pulse basket with total production of 3.38 million tonnes from 4.55 million ha area (Anon., 2019). On account of their value as nutritious food, feed and forage, pulses remained an integral component of subsistence cropping system since time immemorial. Weed is one of the important limiting factor in pigeonpea production through competition for environmental resource and can reduce yield by 30-45 \% (Talnikar et al., 2008). Today, the most of pigeonpea producers use chemical herbicides for weed control in pigeonpea, Application of high dose of chemical herbicide in agricultural production causes many adverse effects that most of them relating to incorrect use of herbicide. One of the adverse effect of herbicides application is evolution of resistant weed biotypes.

The major challenge for India is that of producing adequate food for the ever growing human population. Traditional method of pigeonpea cultivation is no longer a viable system of crop production because of increasing human population on limited arable land, which has reduced the duration of fallow, low soil fertility, declining crop yield and high weed pressure are some of the problems that have been associated with reduced fallow duration and intensification of cropping systems. Pigeonpea being long duration crop, cultivated with wider spacing due to which weed management in these rows plays significant role on yield of the crop. These weeds can cause $20-90 \%$ yield loss in pulse crops (Pooniya et al., 2015). Management of weeds by using herbicide is the commonly followed method. Recent advances in management of weeds through plant water extracts i.e., allelopathy is gaining importance due to better control of weeds, cost effective and biodegradability.

\section{Materials and Methods}

A pot experiment was carried out at Zonal Agriculture Research Station, Kalaburagi (Karnataka), during kharif 2018 and 2019; the study was conducted to study the role of allelopathic weed management on nutrient uptake in pigeonpea (Cajanus cajan L.). The soil was medium deep black with soil $\mathrm{pH}$ (8.13), EC (0.25 dS $\left.\mathrm{m}^{-1}\right)$, available nitrogen $\left(242 \mathrm{~kg} \mathrm{ha}^{-1}\right), \mathrm{P}_{2} \mathrm{O}_{5}\left(34 \mathrm{~kg} \mathrm{ha}^{-1}\right)$ and $\mathrm{K}_{2} \mathrm{O}(347$ $\left.\mathrm{kg} \mathrm{ha}{ }^{-1}\right)$. The experiment was laid out in completely randomised block design which comprised of 18 treatments in three replications, consisting four plant extracts, i.e., Sorghum, Parthenium, Cassia and Eucalyptus and imazythapyre herbicide. All the practices followed were according to package of practice of UAS, Raichur. Treatments as follows; $\mathrm{T}_{1}::$ Imazythapyre @ $18.75 \mathrm{~g}$ a.i./ha + Sorghum plant extract at 2025 DAS, $\mathrm{T}_{2}:$ : Imazythapyre @ $37.5 \mathrm{~g}$ a.i./ha + Sorghum plant extract at 20-25 DAS, $\mathrm{T}_{3}:$ : Imazythapyre: @ 56.25 g a.i./ha + Sorghum plant extract at 20-25 DAS, $\mathrm{T}_{4}:$ : Only Sorghum plant extract at 20-25 DAS, $\mathrm{T}_{5}:$ : Imazythapyre @18.75 g a.i./ha + Parthenium plant extract at 20-25 DAS, $\mathrm{T}_{6}:$ : Imazythapyre @ $37.5 \mathrm{~g}$ a.i./ha + Parthenium plant extract at 20-25 DAS, T $7:$ Imazythapyre: @ $56.25 \mathrm{~g}$ a.i./ha + Parthenium plant extract at 20-25 DAS, $\mathrm{T}_{8}::$ Only Parthenium plant extract at 20-25 DAS, T9:: Imazythapyre @ $18.75 \mathrm{~g}$ a.i./ha + Eucalyptus: leaf extract at 20-25 DAS, $\mathrm{T}_{10}:$ : Imazythapyre @ $37.5 \mathrm{~g}$ a.i./ha + Eucalyptus leaf extract at 20-25 DAS, T $11:$ : Imazythapyre @ $56.25 \mathrm{~g}$ a.i./ha + Eucalyptus leaf extract at 20-25 DAS, $\mathrm{T}_{12}:$ : Only 
Eucalyptus leaf extract at 20-25 DAS, $\mathrm{T}_{13}:$ : Imazythapyre @ $18.75 \mathrm{~g}$ a.i./ha + Cassia plant extract at 20-25 DAS, T14:: Imazythapyre @ $37.5 \mathrm{~g}$ a.i./ha + Cassia plant extract at 20-25 DAS, T $15:$ : Imazythapyre @ 56.25 g a.i./ha + Cassia plant extract at 20-25 DAS, T16:: Only Cassia plant extract at 20-25 DAS, $\mathrm{T}_{17}:$ : Weed free and $\mathrm{T}_{18}:$ : Unweeded check were used in the experiment.

Recommended dose of N, P and K (25:50:00 $\mathrm{kg} \mathrm{N}, \mathrm{P}_{2} \mathrm{O}_{5} \mathrm{~K}_{2} \mathrm{O}$ ha $^{-1}$ ) were applied to the soil in the form of urea, di-ammonium phosphate and muriate of potash at the time of sowing and subsequent applications were done by following package of practice. At each spot two seeds were dibbled up to 4 to $5 \mathrm{~cm}$ deep in the seed lines.

The pigeonpea variety, TS-3R was used during both the years and was sown at spacing of $90 \times 30 \mathrm{~cm}$. All agronomical packages of practices were followed to raise the crop. Nitrogen, phosphorus and potassium content in composite plant sample of pigeopea at harvest was estimated by modified micro-kjeldhal method, vanadomolybdate yellow colour method and flame photometer method, respectively as outlined by (Jackson, 1973).

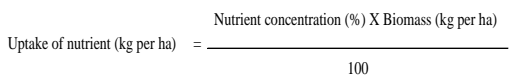

The data of each crop season were statistically analyzed. Fisher's method of analysis of variance was applied for analysis and interpretation of the data as given by Panse and Sukhatme, 1967.

The level of significance used in ' $F$ ' test was at $p=0.05$. Critical difference values were calculated whenever ' $F$ ' was significant. In other cases, values of standard error of means have been provided.

\section{Results and Discussion}

\section{Grain yield ( $\left.\mathrm{kg} \mathrm{ha}^{-1}\right)$}

Grain yield as influenced by allelopatheic effect of plant extracts as weed management practice in pigeonpea is presented in the Table 1 and is clear that it varied significantly in different treatments. Significantly higher grain yield was seen in weed free treatment $\left(2336 \mathrm{~kg} \mathrm{ha}{ }^{-1}\right)$ and was on par with imazythapyre@56.25 g a.i./ha + sorghum plant extract at 20-25 DAS (2175 kg ha'), imazythapyre @ $56.25 \mathrm{~g}$ a.i./ha + cassia plant extract at 20-25 DAS (2054 kg ha ${ }^{-1}$, imazythapyre @ $56.25 \mathrm{~g}$ a.i./ha + parthenium plant extract at 20-25 DAS (2014 kg ha'), imazythapyre @ 37.5 g a.i./ha + sorghum plant extract at 20-25 DAS (1998 kg ha'), imazythapyre@56.25 g a.i./ha + eucalyptus leaf extract at 20-25 DAS (1981 kg ha'), imazythapyre @37.5 g a.i./ha + cassia plant extract at 20-25 DAS (1968 kg ha ${ }^{-1}$ ), imazythapyre@37.5 g a.i./ha + parthenium plant extract at 20-25 DAS (1954 kg ha $\left.{ }^{-1}\right)$ and imazythapyre @ $37.5 \mathrm{~g}$ a.i./ha + eucalyptus leaf extract at 20-25 DAS (1933 kg ha $\left.{ }^{-1}\right)$ and significantly lower grain yield was seen $(964$ $\mathrm{kg} \mathrm{ha}^{-1}$ ) in unweeded check in pooled data.

Extracts and residues of tested species might have the potential to be used for preemergence and post-emergence weed control (Nekonam et al., 2013). Application of reduced dose of herbicides mixed with allelopathic water extracts produced higher grain yield compared with the application of reduced dose of herbicides (Khan and Khan, 2012).

Gilbert et al., (1999) reported that allelochemical facilitate nutrient solubilization and release nutrients from complex forms. Under low phosphorus (P) levels, plant release phosphatases which improve $\mathrm{P}$ availability through hydrolysis. 
Phenolics improve release and uptake of $\mathrm{P}$, Iron $(\mathrm{Fe})$ and other nutrients under their less availability. The basic function is the solublization of nutrients. They make nutrients more mobile and thus improve their uptake in plant body.

Table.1 Grain yield $\left(\mathrm{kg} \mathrm{ha}^{-1}\right)$ as influenced by weed management in pigeonpea by allelopathy in pot culture experiment

\begin{tabular}{|c|c|c|c|}
\hline \multirow[t]{2}{*}{ Treatment } & \multicolumn{3}{|c|}{ Grain yield $\left(\mathrm{kg} \mathrm{ha}^{-1}\right)$} \\
\hline & 2018 & 2019 & Pooled \\
\hline $\begin{array}{l}\mathrm{T}_{1}: \text { Imazythapyre @ } 18.75 \mathrm{~g} \text { a.i./ha + Sorghum } \\
\text { plant extract at 20-25 DAS }\end{array}$ & 1529 & 1752 & 1641 \\
\hline $\begin{array}{l}\text { T2 : Imazythapyre @ } 37.5 \mathrm{~g} \text { a.i./ha + Sorghum } \\
\text { plant extract at 20-25 DAS }\end{array}$ & 1869 & 2126 & 1998 \\
\hline $\begin{array}{l}\mathrm{T}_{3} \text { : Imazythapyre @ } 56.25 \mathrm{~g} \text { a.i./ha + Sorghum } \\
\text { plant extract at 20-25 DAS }\end{array}$ & 2054 & 2296 & 2175 \\
\hline $\mathrm{T}_{4}:$ Only Sorghum plant extract at 20-25 DAS & 1005 & 1407 & 1206 \\
\hline $\begin{array}{l}\mathrm{T}_{5}: \text { Imazythapyre @ } 18.75 \mathrm{~g} \text { a.i./ha }+ \\
\text { Parthenium plant extract at } 20-25 \text { DAS }\end{array}$ & 1355 & 1703 & 1529 \\
\hline $\begin{array}{l}\mathrm{T}_{6} \text { : Imazythapyre @ } 37.5 \mathrm{~g} \text { a.i./ha + Parthenium } \\
\text { plant extract at 20-25 DAS }\end{array}$ & 1827 & 2081 & 1954 \\
\hline $\begin{array}{l}\mathrm{T}_{7}: \text { Imazythapyre @ } 56.25 \mathrm{~g} \text { a.i./ha }+ \\
\text { Parthenium plant extract at } 20-25 \text { DAS }\end{array}$ & 1879 & 2148 & 2014 \\
\hline $\mathrm{T}_{8}$ : Only Parthenium plant extract at 20-25 DAS & 918 & 1333 & 1126 \\
\hline $\begin{array}{l}\text { T9 }_{9} \text { : Imazythapyre @ } 18.75 \mathrm{~g} \text { a.i./ha + Eucalyptus } \\
\text { leaf extract at 20-25 DAS }\end{array}$ & 1311 & 1666 & 1489 \\
\hline $\begin{array}{l}\mathrm{T}_{10} \text { : Imazythapyre @ } 37.5 \mathrm{~g} \text { a.i./ha + Eucalyptus } \\
\text { leaf extract at 20-25 DAS }\end{array}$ & 1792 & 2074 & 1933 \\
\hline $\begin{array}{l}\mathrm{T}_{11}: \text { Imazythapyre @ } 56.25 \mathrm{~g} \text { a.i./ha }+ \\
\text { Eucalyptus leaf extract at } 20-25 \text { DAS }\end{array}$ & 1850 & 2111 & 1981 \\
\hline$T_{12}:$ Only Eucalyptus leaf extract at 20-25 DAS & 1005 & 1400 & 1203 \\
\hline $\begin{array}{l}\mathrm{T}_{13}: \text { Imazythapyre @ } 18.75 \mathrm{~g} \text { a.i./ha + Cassia } \\
\text { plant extract at 20-25 DAS }\end{array}$ & 1398 & 1740 & 1569 \\
\hline $\begin{array}{l}\mathrm{T}_{14} \text { : Imazythapyre @ } 37.5 \mathrm{~g} \text { a.i./ha + Cassia } \\
\text { plant extract at 20-25 DAS }\end{array}$ & 1835 & 2100 & 1968 \\
\hline $\begin{array}{l}\text { T}_{15} \text { : Imazythapyre @ } 56.25 \text { g a.i./ha + Cassia } \\
\text { plant extract at 20-25 DAS }\end{array}$ & 1923 & 2185 & 2054 \\
\hline$T_{16}$ : Only Cassia plant extract at 20-25 DAS & 961 & 1370 & 1166 \\
\hline$T_{17}:$ Weed free & 2228 & 2444 & 2336 \\
\hline $\mathbf{T}_{18}:$ Unweeded check & 743 & 1185 & 964 \\
\hline S.Em \pm & 129 & 150 & 111 \\
\hline C.D. at $5 \%$ & 494 & 577 & 428 \\
\hline
\end{tabular}


Table.2 Nitrogen $\left(\mathrm{kg} \mathrm{ha}^{-1}\right)$ uptake as influenced by weed management in pigeonpea by allelopathy in pot culture experiment

\begin{tabular}{|c|c|c|c|}
\hline \multirow[t]{2}{*}{ Treatment } & \multicolumn{3}{|c|}{ Nitrogen $\left(\mathrm{kg} \mathrm{ha}^{-1}\right)$} \\
\hline & 2018 & 2019 & Pooled \\
\hline $\begin{array}{l}\mathrm{T}_{1} \text { : Imazythapyre @ } 18.75 \mathrm{~g} \text { a.i./ha + Sorghum } \\
\text { plant extract at 20-25 DAS }\end{array}$ & 94.28 & 98.99 & 96.64 \\
\hline $\begin{array}{l}\mathrm{T}_{2} \text { : Imazythapyre @ } 37.5 \mathrm{~g} \text { a.i./ha + Sorghum } \\
\text { plant extract at 20-25 DAS }\end{array}$ & 99.48 & 104.42 & 101.95 \\
\hline $\begin{array}{l}\mathrm{T}_{3} \text { : Imazythapyre @ } 56.25 \mathrm{~g} \text { a.i./ha + Sorghum } \\
\text { plant extract at 20-25 DAS }\end{array}$ & 107.21 & 112.59 & 109.90 \\
\hline $\mathrm{T}_{4}:$ Only Sorghum plant extract at 20-25 DAS & 89.21 & 93.69 & 91.45 \\
\hline $\begin{array}{l}\mathrm{T}_{5}: \text { Imazythapyre @ } 18.75 \mathrm{~g} \text { a.i./ha }+ \\
\text { Parthenium plant extract at } 20-25 \text { DAS }\end{array}$ & 92.10 & 96.70 & 94.40 \\
\hline $\begin{array}{l}\mathrm{T}_{6} \text { : Imazythapyre @ } 37.5 \mathrm{~g} \text { a.i./ha + Parthenium } \\
\text { plant extract at 20-25 DAS }\end{array}$ & 97.10 & 101.99 & 99.54 \\
\hline $\begin{array}{l}\mathrm{T}_{7}: \text { Imazythapyre } @ 56.25 \mathrm{~g} \text { a.i./ha }+ \\
\text { Parthenium plant extract at } 20-25 \mathrm{DAS}\end{array}$ & 100.15 & 105.19 & 102.67 \\
\hline$T_{8}$ : Only Parthenium plant extract at 20-25 DAS & 87.80 & 92.19 & 90.00 \\
\hline $\begin{array}{l}\mathrm{T}_{9} \text { : Imazythapyre @ } 18.75 \mathrm{~g} \text { a.i./ha + Eucalyptus } \\
\text { leaf extract at 20-25 DAS }\end{array}$ & 91.35 & 95.91 & 93.63 \\
\hline $\begin{aligned} \mathrm{T}_{10}: & \text { Imazythapyre @ } 37.5 \mathrm{~g} \text { a.i./ha + Eucalyptus } \\
& \text { leaf extract at 20-25 DAS }\end{aligned}$ & 96.30 & 101.12 & 98.71 \\
\hline $\begin{array}{l}\mathrm{T}_{11}: \text { Imazythapyre @ } 56.25 \mathrm{~g} \text { a.i./ha }+ \\
\text { Eucalyptus leaf extract at 20-25 DAS }\end{array}$ & 98.12 & 103.03 & 100.57 \\
\hline$T_{12}:$ Only Eucalyptus leaf extract at 20-25 DAS & 89.10 & 93.56 & 91.33 \\
\hline $\begin{array}{l}\mathrm{T}_{13} \text { : Imazythapyre @ } 18.75 \mathrm{~g} \text { a.i./ha + Cassia } \\
\text { plant extract at 20-25 DAS }\end{array}$ & 93.55 & 98.23 & 95.89 \\
\hline $\begin{array}{l}\mathrm{T}_{14} \text { : Imazythapyre @ } 37.5 \mathrm{~g} \text { a.i./ha + Cassia } \\
\text { plant extract at 20-25 DAS }\end{array}$ & 97.95 & 102.85 & 100.40 \\
\hline $\begin{array}{l}\mathrm{T}_{15} \text { : Imazythapyre @ } 56.25 \mathrm{~g} \text { a.i./ha + Cassia } \\
\text { plant extract at 20-25 DAS }\end{array}$ & 101.33 & 106.40 & 103.86 \\
\hline$T_{16}:$ Only Cassia plant extract at 20-25 DAS & 88.50 & 92.93 & 90.71 \\
\hline$T_{17}:$ Weed free & 109.20 & 114.69 & 111.94 \\
\hline$T_{18}:$ Unweeded check & 85.11 & 89.37 & 87.24 \\
\hline S.Em \pm & 3.62 & 3.85 & 3.64 \\
\hline C.D. at $5 \%$ & 13.93 & 14.79 & 14.01 \\
\hline
\end{tabular}


Table.3 Phosphorous $\left(\mathrm{kg} \mathrm{ha}^{-1}\right)$ uptake as influenced by weed management in pigeonpea by allelopathy in pot culture experiment

\begin{tabular}{|c|c|c|c|}
\hline \multirow[t]{2}{*}{ Treatment } & \multicolumn{3}{|c|}{ Phosphorous (kg ha $\left.{ }^{-1}\right)$} \\
\hline & 2018 & 2019 & Pooled \\
\hline $\begin{array}{c}\mathrm{T}_{1}: \text { Imazythapyre @ } 18.75 \mathrm{~g} \text { a.i./ha + Sorghum } \\
\text { plant extract at 20-25 DAS }\end{array}$ & 29.45 & 32.10 & 30.78 \\
\hline $\begin{array}{l}\mathrm{T}_{2} \text { : Imazythapyre @ } 37.5 \mathrm{~g} \text { a.i./ha + Sorghum plant } \\
\text { extract at 20-25 DAS }\end{array}$ & 34.10 & 37.17 & 35.63 \\
\hline $\begin{array}{l}\mathrm{T}_{3}: \text { Imazythapyre } @ 56.25 \mathrm{~g} \text { a.i./ha + Sorghum } \\
\text { plant extract at 20-25 DAS }\end{array}$ & 36.80 & 40.11 & 38.46 \\
\hline $\mathrm{T}_{4}:$ Only Sorghum plant extract at 20-25 DAS & 23.85 & 26.00 & 24.92 \\
\hline $\begin{array}{l}\mathrm{T}_{5}: \text { Imazythapyre @ } 18.75 \text { g a.i./ha + Parthenium } \\
\text { plant extract at 20-25 DAS }\end{array}$ & 26.70 & 29.10 & 27.90 \\
\hline $\begin{array}{l}\mathrm{T}_{6} \text { : Imazythapyre @ } 37.5 \mathrm{~g} \text { a.i./ha + Parthenium } \\
\text { plant extract at 20-25 DAS }\end{array}$ & 32.13 & 35.02 & 33.58 \\
\hline $\begin{array}{l}\mathrm{T}_{7}: \text { Imazythapyre @ } 56.25 \mathrm{~g} \text { a.i./ha + Parthenium } \\
\text { plant extract at 20-25 DAS }\end{array}$ & 35.25 & 38.42 & 36.84 \\
\hline $\mathrm{T}_{8}$ : Only Parthenium plant extract at 20-25 DAS & 24.22 & 26.40 & 25.31 \\
\hline $\begin{array}{l}\text { T9: Imazythapyre @ } 18.75 \text { g a.i./ha + Eucalyptus leaf } \\
\text { extract at 20-25 DAS }\end{array}$ & 26.11 & 28.46 & 27.28 \\
\hline $\begin{aligned} \mathrm{T}_{10}: & \text { Imazythapyre @ } 37.5 \mathrm{~g} \text { a.i./ha + Eucalyptus } \\
& \text { leaf extract at 20-25 DAS }\end{aligned}$ & 31.30 & 34.12 & 32.71 \\
\hline $\begin{array}{l}\mathrm{T}_{11} \text { : Imazythapyre @ } 56.25 \mathrm{~g} \text { a.i./ha + Eucalyptus } \\
\text { leaf extract at 20-25 DAS }\end{array}$ & 33.45 & 36.46 & 34.96 \\
\hline$T_{12}:$ Only Eucalyptus leaf extract at 20-25 DAS & 23.80 & 25.94 & 24.87 \\
\hline $\begin{array}{l}T_{13}: \text { Imazythapyre @ } 18.75 \text { g a.i./ha + Cassia plant } \\
\text { extract at 20-25 DAS }\end{array}$ & 27.30 & 29.76 & 28.53 \\
\hline $\begin{array}{c}\mathrm{T}_{14} \text { : Imazythapyre @ } 37.5 \mathrm{~g} \text { a.i./ha + Cassia plant } \\
\text { extract at 20-25 DAS }\end{array}$ & 33.38 & 36.38 & 34.88 \\
\hline $\begin{array}{l}\mathrm{T}_{15} \text { : Imazythapyre @ } 56.25 \mathrm{~g} \text { a.i./ha + Cassia plant } \\
\text { extract at 20-25 DAS }\end{array}$ & 36.12 & 39.37 & 37.75 \\
\hline$T_{16}$ : Only Cassia plant extract at 20-25 DAS & 24.90 & 27.14 & 26.02 \\
\hline$T_{17}:$ Weed free & 37.65 & 41.01 & 39.33 \\
\hline $\mathbf{T}_{18}:$ Unweeded check & 22.35 & 24.36 & 23.36 \\
\hline S.Em \pm & 1.73 & 1.90 & 1.81 \\
\hline C.D. at $5 \%$ & 6.66 & 7.29 & 6.94 \\
\hline
\end{tabular}


Table.4 Potassium $\left(\mathrm{kg} \mathrm{ha}^{-1}\right)$ uptake as influenced by weed management in pigeonpea by allelopathy in pot culture experiment

\begin{tabular}{|c|c|c|c|}
\hline \multirow[t]{2}{*}{ Treatment } & \multicolumn{3}{|c|}{ Potassium $\left(\mathrm{kg} \mathrm{ha}^{-1}\right)$} \\
\hline & 2018 & 2019 & Pooled \\
\hline $\begin{array}{l}\mathrm{T}_{1}: \text { Imazythapyre @ } 18.75 \mathrm{~g} \text { a.i./ha + Sorghum } \\
\text { plant extract at 20-25 DAS }\end{array}$ & 38.65 & 41.74 & 40.20 \\
\hline $\begin{array}{l}\mathrm{T}_{2} \text { : Imazythapyre @ } 37.5 \mathrm{~g} \text { a.i./ha + Sorghum } \\
\text { plant } \\
\quad \text { extract at 20-25 DAS }\end{array}$ & 42.16 & 45.53 & 43.85 \\
\hline $\begin{array}{l}\mathrm{T}_{3}: \text { Imazythapyre @ } 56.25 \mathrm{~g} \text { a.i./ha + Sorghum } \\
\text { plant extract at 20-25 DAS }\end{array}$ & 44.80 & 48.38 & 46.59 \\
\hline$T_{4}:$ Only Sorghum plant extract at 20-25 DAS & 34.33 & 37.08 & 35.70 \\
\hline $\begin{array}{l}\mathrm{T}_{5} \text { : Imazythapyre @ } 18.75 \mathrm{~g} \text { a.i./ha + Parthenium } \\
\text { plant extract at 20-25 DAS }\end{array}$ & 36.45 & 39.37 & 37.91 \\
\hline $\begin{array}{l}\mathrm{T}_{6} \text { : Imazythapyre @ } 37.5 \mathrm{~g} \text { a.i./ha + Parthenium } \\
\text { plant extract at 20-25 DAS }\end{array}$ & 40.82 & 44.09 & 42.45 \\
\hline $\begin{array}{l}\mathrm{T}_{7}: \text { Imazythapyre @ } 56.25 \mathrm{~g} \text { a.i./ha }+ \\
\text { Parthenium plant extract at } 20-25 \mathrm{DAS}\end{array}$ & 42.72 & 46.14 & 44.43 \\
\hline$T_{8}:$ Only Parthenium plant extract at 20-25 DAS & 32.10 & 34.67 & 33.38 \\
\hline $\begin{array}{l}\text { T9: Imazythapyre @ } 18.75 \text { g a.i./ha + Eucalyptus } \\
\text { leaf extract at } 20-25 \text { DAS }\end{array}$ & 36.02 & 38.90 & 37.46 \\
\hline $\begin{array}{l}\mathrm{T}_{10} \text { : Imazythapyre @ } 37.5 \mathrm{~g} \text { a.i./ha + Eucalyptus } \\
\text { leaf extract at 20-25 DAS }\end{array}$ & 40.06 & 43.26 & 41.66 \\
\hline $\begin{array}{l}\mathrm{T}_{11} \text { : Imazythapyre @ } 56.25 \mathrm{~g} \text { a.i./ha + Eucalyptus } \\
\text { leaf extract at 20-25 DAS }\end{array}$ & 41.50 & 44.82 & 43.16 \\
\hline$T_{12}:$ Only Eucalyptus leaf extract at 20-25 DAS & 34.21 & 36.95 & 35.58 \\
\hline $\begin{array}{l}\mathrm{T}_{13}: \text { Imazythapyre @ } 18.75 \mathrm{~g} \text { a.i./ha + Cassia } \\
\text { plant extract at } 20-25 \text { DAS }\end{array}$ & 37.12 & 40.09 & 38.60 \\
\hline $\begin{array}{l}\mathrm{T}_{14} \text { : Imazythapyre @ } 37.5 \mathrm{~g} \text { a.i./ha + Cassia plant } \\
\text { extract at 20-25 DAS }\end{array}$ & 41.32 & 44.63 & 42.97 \\
\hline $\begin{array}{l}\mathrm{T}_{15}: \text { Imazythapyre @ } 56.25 \mathrm{~g} \text { a.i./ha + Cassia } \\
\text { plant extract at 20-25 DAS }\end{array}$ & 43.55 & 47.03 & 45.29 \\
\hline$T_{16}:$ Only Cassia plant extract at $20-25$ DAS & 33.65 & 36.34 & 35.00 \\
\hline$T_{17}:$ Weed free & 46.65 & 50.38 & 48.52 \\
\hline $\mathbf{T}_{18}:$ Unweeded check & 30.90 & 33.37 & 32.14 \\
\hline S.Em \pm & 1.82 & 2.16 & 1.93 \\
\hline C.D. at $5 \%$ & 6.98 & 8.32 & 7.41 \\
\hline
\end{tabular}


Fig.1 Nutrients uptake $\left(\mathrm{kg} \mathrm{ha}^{-1}\right)$ by pigeonpea crop as influenced by weed management in pigeonpea by allelopathy in pot culture experiment

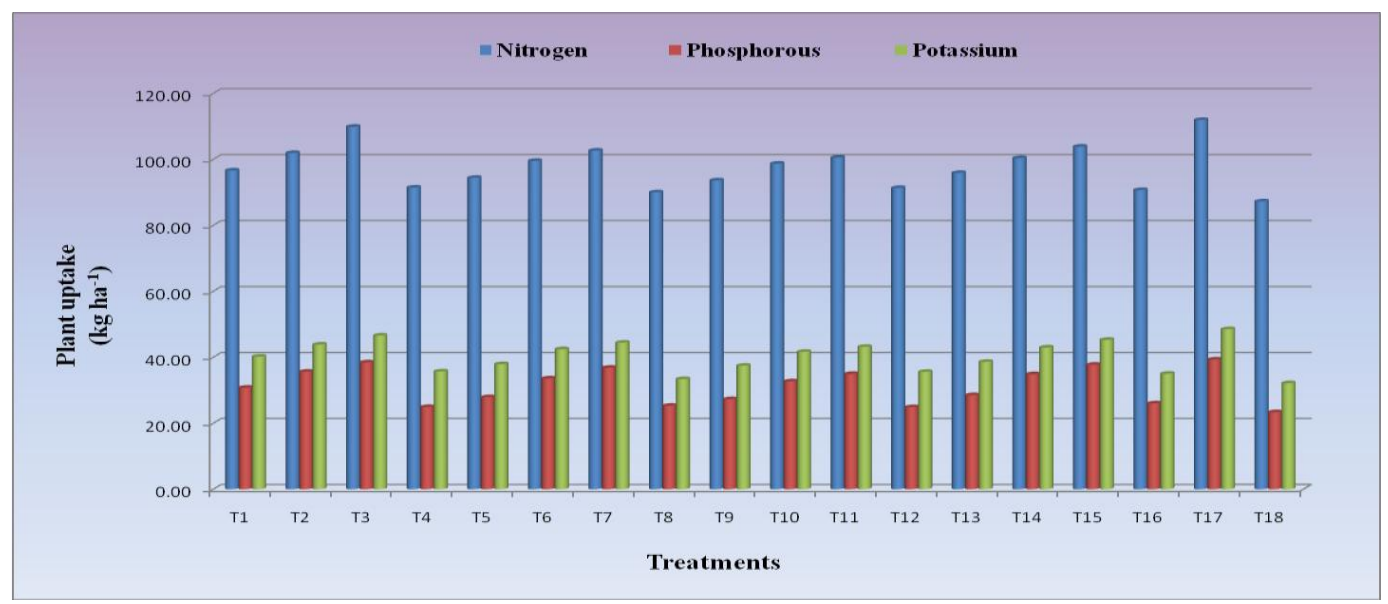

Nitrogen uptake $\left(\mathrm{kg} \mathrm{ha}^{-1}\right)$

Nitrogen uptake data as influenced by allelopatheic effect of plant extracts as weed management practice in pigeonpea is presented in Table 2 and Fig. 1 and it varied significantly among different weed management practices. Significantly higher nitrogen uptake was noted in weed free treatment $\left(111.94 \mathrm{~kg} \mathrm{ha}^{-1}\right)$ in pooled data and other treatments like; imazythapyre @ 56.25 g a.i./ha + sorghum plant extract at 20-25 DAS (109.90 kg ha ${ }^{-1}$ ), imazythapyre @ 56.25 g a.i./ha + cassia plant extract at 20-25 DAS (103.86 kg ha ${ }^{-1}$ ), imazythapyre @ $56.25 \mathrm{~g}$ a.i./ha + parthenium plant extract at 20-25 DAS (102.67 kg ha ${ }^{-1}$ ), imazythapyre @ $37.5 \mathrm{~g}$ a.i./ha + sorghum plant extract at 20-25 DAS (101.95 kg ha-1), imazythapyre @ $56.25 \mathrm{~g}$ a.i./ha + eucalyptus leaf extract at 20-25 DAS (100.57 kg ha $\left.{ }^{-1}\right)$, imazythapyre @ $37.5 \mathrm{~g}$ a.i./ha + cassia plant extract at 20-25 DAS (100.40 kg ha ${ }^{-1}$ ), imazythapyre @ $37.5 \mathrm{~g}$ a.i./ha + parthenium plant extract at 20-25 DAS (99.54 kg ha ${ }^{-1}$ ) and imazythapyre @ $37.5 \mathrm{~g}$ a.i./ha + eucalyptus leaf extract at 2025 DAS (98.71 kg ha ${ }^{-1}$ ) were on par with it and significantly lower nitrogen uptake was seen $\left(87.24 \mathrm{~kg} \mathrm{ha}^{-1}\right)$ in unweeded check.
Phosphorous uptake (kg ha $\left.{ }^{-1}\right)$

The data pertaining to phosphorous uptake as influenced by plant extracts as weed management practice in pigeonpea by allelopathy is presented (Table 3 and Fig. 1) and it varied significantly among different weed management practices. The treatments; imazythapyre @ $56.25 \mathrm{~g}$ a.i./ha + sorghum plant extract at 20-25 DAS (38.46 kg ha-1), imazythapyre @ $56.25 \mathrm{~g}$ a.i./ha + cassia plant extract at 20-25 DAS (37.75kg ha $\left.\mathrm{kg}^{-1}\right)$, imazythapyre@56.25 g a.i./ha + parthenium plant extract at 20-25 DAS (36.84 kg ha-1), imazythapyre@ 37.5 g a.i./ha + sorghum plant extract at 20-25 DAS (35.63 kg ha $\mathrm{kg}^{-1}$, imazythapyre @ $56.25 \mathrm{~g}$ a.i./ha + eucalyptus leaf extract at 20-25 DAS (34.96 kg ha-1), imazythapyre@37.5 g a.i./ha + cassia plant extract at 20-25 DAS (34.88 kg ha-1), imazythapyre@37.5 g a.i./ha + parthenium plant extract at 20-25 DAS (33.5 $\left.\mathrm{kg} \mathrm{ha}^{-1}\right)$ and imazythapyre@37.5 g a.i./ha + eucalyptus leaf extract at 20-25 DAS (32.71 kg ha $\left.{ }^{-1}\right)$ were on par with weed free treatment $(39.33$ $\mathrm{kg} \mathrm{ha}{ }^{-1}$ ) that remarked significantly higher phosphorous uptake and unweeded check recorded significantly lower phosphorous uptake $\left(23.36 \mathrm{~kg} \mathrm{ha}^{-1}\right)$. 


\section{Potassium uptake $\left(\mathrm{kg} \mathrm{ha}^{-1}\right)$}

The potassium uptake data was influenced by plant extracts as weed management practice in pigeonpea by allelopathy and showed that it varied significantly among different weed management practices (Table 4 and Fig. 1). Weed free treatment noted significantly higher potassium uptake $\left(48.52 \mathrm{Kg} \mathrm{ha}^{-1}\right)$ in both the years and in pooled data. Whereas, imazythapyre @ $56.25 \mathrm{~g}$ a.i./ha + sorghum plant extract at 20-25 DAS (46.59 $\left.\mathrm{kg} \mathrm{ha}^{-1}\right)$, imazythapyre @ 56.25 g a.i./ha + cassia plant extract at 20-25 DAS (45.29 $\left.\mathrm{kg} \mathrm{ha}^{-1}\right)$, imazythapyre @ $56.25 \mathrm{~g}$ a.i./ha + parthenium plant extract at 20-25 DAS (44.43 $\left.\mathrm{kg} \mathrm{ha}^{-1}\right)$, imazythapyre @37.5 g a.i./ha + sorghum plant extract at 20-25 DAS (43.85 kg ha $\left.{ }^{-1}\right)$, imazythapyre @ $56.25 \mathrm{~g}$ a.i./ha + eucalyptus leaf extract at 20-25 DAS (43.16 kg ha $\left.{ }^{-1}\right)$, imazythapyre @ $37.5 \mathrm{~g}$ a.i./ha + cassia plant extract at 20-25 DAS (42.97 kg ha $\left.{ }^{-1}\right)$, imazythapyre @ $37.5 \mathrm{~g}$ a.i./ha + parthenium plant extract at 20-25 DAS (42.45 $\left.\mathrm{kg} \mathrm{ha}^{-1}\right)$ and imazythapyre @ $37.5 \mathrm{~g}$ a.i./ha + eucalyptus leaf extract at 20-25 DAS (41.66 $\mathrm{kg} \mathrm{ha}^{-1}$ ) were on par with it and unweeded check recorded significantly lower potassium uptake $\left(32.14 \mathrm{~kg} \mathrm{ha}^{-1}\right)$.

Weed management practices significantly influenced the nutrient uptake by crop plants $(\mathrm{N}, \mathrm{P}$ and $\mathrm{K})$ among all the treatments after harvest of pigeonpea. Imazythapyre @ 56.25 g a.i./ha + sorghum plant extract at 20-25 DAS, imazythapyre @ 56.25 g a.i./ha + cassia plant extract at 20-25 DAS, imazythapyre @ $56.25 \mathrm{~g}$ a.i./ha + parthenium plant extract at 20-25 DAS, imazythapyre @ 37.5 g a.i./ha + sorghum plant extract at 20-25 DAS, imazythapyre @ $56.25 \mathrm{~g}$ a.i./ha + eucalyptus leaf extract at 20-25 DAS, imazythapyre @ $37.5 \mathrm{~g}$ a.i./ha + cassia plant extract at 20-25 DAS, imazythapyre @ 37.5 g a.i./ha + parthenium plant extract at 20-25 DAS and imazythapyre@37.5g a.i./ha + eucalyptus leaf extract at 20-25 DAS recorded significantly higher uptake of nitrogen, phosphorus and potassium compared to rest of the treatments which might be due to higher biomass in these treatments and therefore higher uptake of nutrients observed.

Allelochemicals have been reported to decrease uptake of mineral elements by altering the functions of plasma membranes in plant roots, depolarizing the electrochemical potential gradient across membranes; a primary driving force for ion uptake and decreasing ATP content of cells by inhibiting electron transport and oxidative phosphorylation and by changing the permeability of membranes to mineral ions (Balke 1985). Ferulic acid is an allelochemical found in many plants such as sorghum and rice which is also exuded into soil by these plants. Booker et al., (1992) used intact cucumber (Cucumis sativus) seedlings to evaluate the effect of ferulic acid on mineral uptake and water relations of cucumber and observed that ferulic acid inhibited mineral uptake, especially $\mathrm{N}$ and also reduced leaf: turgor pressure and water potential.

Allelochemicals, upon release into the rhizosphere may influence nutrient movement, availability and uptake by plants. Changes in microbial activities and nutrient dynamics on addition of allelochemicals to the soil have been reported (Karmarkar and Tabatabai 1991). Usually, allelochemicals are first perceived by the receiver plant's roots which may then affect nutrient uptake and these compounds may restrict or improve the nutrients mobility to plants ( $\mathrm{Yu}$ and Matsui 1997). Dalipu (2001) studied the effect of different weed species on leaf total chlorophyll content and leaf nitrate reductase (NR) activity of rice plant.: They reported that both the weeds Cynadon dactylon and Cyperus rotundus at low concentration (1:20) 
increased the total chlorophyll content, nitrate reductase (NR) activity of rice plant, uptake of $\mathrm{N}, \mathrm{P}, \mathrm{K}$ at harvest and soil available $\mathrm{N}, \mathrm{P}$ and $\mathrm{K}$ were low at 25 and $50 \mathrm{DAS}$, it might be due to growth promotion property of allelopathic water extracts of these weeds at low concentration.

Sorghum, Parthenium, Cassia and Eucalyptus plant extracts with imazythapyre herbicide at $50 \%$ and $75 \%$ help to get good control of weeds in pigeonpea. Thereby help to improve the growth of pigeonpea indirectly by good uptake of the available nutrients in the soil, less competition for the resources from weeds and through releasing allelochemicals directly into crop rhizosphere that help to mineralize the unavailable form of nutrient and improve the growth of the crop directly. Even the use of these plant extracts is cost effective which reduces the burden of farmers with respect to herbicides. Hence plant extracts can be used with herbicide which will help to reduce the usage of herbicide by $50 \%$ and dependency on herbicides can be reduced, through which ill effects of herbicides on soil can be avoided, soil fertility can be maintained for longer period and this helps improve the crop growth.

\section{References}

Anonymous, 2019 https://www.indiastat.com/table/agric ulture-data/2/arha-tur/19566/17337/ data.aspx.

Balke, N. E., 1985, Effects of allelochemicals on mineral uptake and associated physiological processes. In: Thompson AC (ed) The chemistry of allelopathy: biochemical interactions among plants. American Bio. Soc., pp: 161178.

Booker, F. L., Blum, U., Fiscus, E. L., 1992, Short-term effect of ferulic acid on ion uptake and water relations in cucumber seedlings. J. Exp. Bot., 43: 649-655.

Dalipu, K., 2001, Allelopathic effect of weeds on physio-chemical properties of rice and nutrient status of soil. Ecol. Env. and Cons., 7(1): 79-85.

Gilbert, G. A., Knight, J. D., Allan, D. L. and Vance, C. P., 1999, Acid phosphatase activity in phosphorus deficient white lupin roots. Plant Cell Environ., 22: 801-810.

Jackson, M. L., 1973, Soil Chemical Analysis. Oxford IBH Publishing House, Bombay. p. 38.

Karmarkar, S. V. and Tabatabai, M. A., 1991, Effects of biotechnology byproducts and organic acids on nitrification in soils. Biol. Fertil. Soils., 12: 165-169.

Khan, R. and Khan, A. M., 2012, Weed control efficiency of bioherbicides and their impact on grain yield of wheat (Triticum aestivum L.). European J. Appl. Sci., 4(5): 216-219.

Nekonam, M. S., Jamshid, R., Hasan, K., Bahram, S., Hajar, A. and Frouzan, B., 2013, Assessment of some medicinal plants for their allelopathic potential against redroot pigweed (Amaranthus retroflexus). J. Plant Prot. Res., 54(1): 90-95.

Panse, V. G. and Sukhatme, P. V., 1967, Statistical Methods for Agricultural Workers, Indian Council of Agricultural Research, New Delhi.

Pooniya, V., Choudhary, A. K., Dass, A., Bana, R. S., Rana, K. S., Rana, D. S., Tyagi, V. K. and Puniya, M. M., 2015, Improved crop management practices for sustainable pulse production: An Indian perspective. Indian J. Agric. Sci., 85(6): 747-758.

Subbaiah, B. Y. and Asija, G. L., 1956, A rapid procedure for the estimation of available nitrogen in soils. Curr. Sci., 25: 259-260.

Talnikar, A. S., Kadam, D. R., Karande, D. R. 
and Jogdand, P. B., 2008, Integrated weed management in pigeonpea [Cajanus cajan (L.) Millsp.]. Int. J. Agric. Sci., 4: 363-370. exudates of cucumber (Cucumis sativus) and allelochemicals on ion uptake by cucumber seedlings. $J$. Chem. Ecol., 23: 817-827.

Yu, J. Q. and Matsui, Y., 1997, Effects of root

\section{How to cite this article:}

Shridhara, B. Nagoli, B. M. Dodamani, M. Y. Ajaykumar, Pandit S. Rathod, Mahadeva Swamy and Basavaraj, K. 2021. Role of Allelopathic Weed Management on Nutrient Uptake in Pigeonpea (Cajanus cajan L.). Int.J.Curr.Microbiol.App.Sci. 10(01): 2019-2029.

doi: https://doi.org/10.20546/ijcmas.2021.1001.233 